\title{
ON $p$-ADIC PERIODS FOR MIXED TATE MOTIVES OVER A NUMBER FIELD
}

\author{
Andre Chatzistamatiou and Sinan Ünver
}

\begin{abstract}
For a number field, we have a Tannaka category of mixed Tate motives at our disposal. We construct $p$-adic points of the associated Tannaka group by using $p$-adic Hodge theory. Extensions of two Tate objects yield functions on the Tannaka group, and we show that evaluation at our $p$-adic points is essentially given by the inverse of the Bloch-Kato exponential map.
\end{abstract}

\section{Contents}

Introduction $\quad 825$

1. Filtered $\phi$-modules and mixed Tate filtered $\phi$-modules 826

1.1. $\quad$ Mixed Tate filtered $\phi$-modules $\quad 826$

1.2. The crystalline logarithmic point $\quad 830$

1.3. The semistable logarithmic point 833

2. Mixed Tate motives over a number field and logarithmic points 838

2.1. $\quad$ Mixed Tate motives $\quad 838$

2.2. Crystalline characterization of unramified motives 840

2.3. Main theorem 841

$\begin{array}{lr}\text { Acknowledgments } & 843\end{array}$

$\begin{array}{ll}\text { References } & 844\end{array}$

\section{Introduction}

For a number field $E$, one has an abelian category of mixed Tate motives $M T(E)$ [3]. A mixed Tate motive comes equipped with a weight filtration $W$, and the associated graded pieces are sums of Tate objects. There is a natural fibre functor $\omega$ defined by

$$
\omega(M)=\bigoplus_{n \in \mathbb{Z}} \operatorname{Hom}\left(\mathbb{Q}(n), \operatorname{gr}_{-2 n}^{W}(M)\right) ;
$$

we denote by $G_{\omega}$ the corresponding Tannaka group.

If $\mathcal{O}$ denotes the ring of integers of $E$ and $x \in \operatorname{Spec}(\mathcal{O})$ is a closed point, then Deligne and Goncharov construct a Tannaka subcategory $M T\left(\mathcal{O}_{x}\right)$ of $M T(E)$ consisting of motives which are unramified at $x[3,1.6]$. We will denote its group of tensor automorphisms by $G_{x}$.

To a mixed Tate motive $M$ we can attach its $p$-adic realization $M_{p}$ which is a representation of the Galois group of $E$ with coefficients in $\mathbb{Q}_{p}$. Suppose that the

Received by the editors October 26, 2011. 
point $x$ lies over the prime $p$, and $\iota: \bar{E} \rightarrow \bar{E}_{x}$ is an imbedding of the algebraic closure of $E$ to that of the completion of $E$ at $x$. This induces a homomorphism between the Galois groups, and restricting the Galois representation $M_{p}$ via this homomorphism gives us a representation of the Galois group of $E_{x}$, which we denote by $M_{\iota, p}$. We will show that $M_{\iota, p}$ is always semistable. Furthermore, $M_{\iota, p}$ is crystalline if and only if $M$ is unramified at $x$, i.e., $M \in M T\left(\mathcal{O}_{x}\right)$ (Theorem 2.2.3). In fact, $p$-adic representations attached to mixed Tate motives are contained in an abelian subcategory which admits a fibre functor $\tau$ similar to $\omega$. Denoting by $H_{\tau}$ the corresponding Tannaka group over $\mathbb{Q}_{p}, p$-adic realization yields a group homomorphism

$$
H_{\tau} \rightarrow G_{\omega} \otimes_{\mathbb{Q}} \mathbb{Q}_{p}
$$

The main purpose of this paper is to construct an $E_{x, s t}$-valued point $\eta_{s t}$ of $H_{\tau}$, where $\operatorname{Spec}\left(E_{x, s t}\right)$ is a one-dimensional affine space over the field $E_{x}$. The $E_{x}$-valued points of $\operatorname{Spec}\left(E_{x, s t}\right)$ correspond naturally to the extensions of the canonical logarithm $\log : \mathcal{O}_{E_{x}}^{\times} \rightarrow E$ to $E^{\times}$. Therefore, any choice of such an extension induces via $\eta_{s t}$ an $E_{x}$-valued point of $H_{\tau}$ and $G_{\omega}$. For the Tannaka subcategory of crystalline representations the picture is simpler: if $H_{\tau, \text { cris }}$ denotes their Tannaka group and $\pi: H_{\tau} \rightarrow H_{\tau, \text { cris }}$ is the projection, then $\pi \circ \eta_{s t}$ factors through $\operatorname{Spec}\left(E_{x}\right)$ and we obtain an $E_{x}$-valued point $\eta$ of $H_{\tau, \text { cris }}$. We denote by $\eta_{x}^{u r}$ the image of $\eta$ in $G_{x}$.

To state our main theorem, we need to recall how extensions $M$ of $\mathbb{Q}(0)$ by $\mathbb{Q}(n)$ in $M T\left(\mathcal{O}_{x}\right)$ give rise to functions on $G_{x}$ for $n \geq 1$. The natural isomorphisms $\alpha: \mathbb{Q} \rightarrow$ $\operatorname{Hom}\left(\mathbb{Q}(n), \operatorname{gr}_{-2 n}^{W} M\right)$ and $\beta: \operatorname{Hom}\left(\mathbb{Q}(0), \operatorname{gr}_{0}^{W} M\right) \rightarrow \mathbb{Q}$ induce elements $\alpha^{-1} \in \omega(M)^{\vee}$ and $\beta^{-1} \in \omega(M)$; we set $M\left(\eta_{x}^{u r}\right)=\alpha^{-1}\left(\eta_{x}^{u r} \cdot \beta^{-1}(1)\right)$.

Theorem (Theorem 2.3.3). For all $n \geq 1$, the map

$$
\operatorname{Ext}_{M T\left(\mathcal{O}_{x}\right)}^{1}(\mathbb{Q}(0), \mathbb{Q}(n)) \rightarrow E_{x}, \quad M \mapsto M\left(\eta_{x}^{u r}\right),
$$

is the composition of the p-adic realization

$$
\operatorname{Ext}_{M T\left(\mathcal{O}_{x}\right)}^{1}(\mathbb{Q}(0), \mathbb{Q}(n)) \rightarrow \operatorname{Ext}_{\text {crys }}^{1}\left(\mathbb{Q}_{p}(0), \mathbb{Q}_{p}(n)\right)
$$

and the inverse of the Bloch-Kato exponential map (2.3.1).

\section{Filtered $\phi$-modules and mixed Tate filtered $\phi$-modules}

\subsection{Mixed Tate filtered $\phi$-modules.}

1.1.1. Let $K$ be a $p$-adic field with residue field $k$, i.e., $\operatorname{char}(K)=0, K$ is complete with respect to a fixed discrete valuation and the residue field $k$ is perfect of characteristic $p$. Let $W(k)$ be the ring of Witt vectors of $k, \sigma: W(k) \rightarrow W(k)$ the Frobenius lift and $K_{0}$ the field of fractions of $W(k)$.

1.1.2. We denote by $M F_{K}^{\phi}$ the category of filtered $\phi$-modules, i.e., the objects are triples $(M, \phi, F)$, where $(M, \phi)$ is an isocrystal over $K_{0}$ and $F$ is a descending, exhaustive and separated filtration on $M_{K}=M \otimes_{K_{0}} K$. We denote by $M F_{K}^{\phi, N}$ the category of filtered $(\phi, N)$-modules, i.e., objects are tuples $(M, \phi, N, F)$ with $(M, \phi, F) \in M F_{K}^{\phi}$ and $N: M \rightarrow M$ is a $K_{0}$-linear endomorphism such that $N \phi=p \phi N$. We consider $M F_{K}^{\phi}$ as full subcategory of $M F_{K}^{\phi, N}$ via the functor $(M, \phi, F) \mapsto(M, \phi, 0, F)$. 
The Dieudonné-Manin classification [4, II, Section 4.1] implies, by descent, that every isocrystal $(M, \phi)$ over $K_{0}$ admits a slope decomposition

$$
M=\bigoplus_{\lambda \in \mathbb{Q}} M_{\lambda}
$$

with $\phi\left(M_{\lambda}\right)=M_{\lambda}$ and $\left(M_{\lambda},\left.\phi\right|_{M_{\lambda}}\right)$ is isoclynic of slope $\lambda$. From the relation $N \phi=$ $p \phi N$, it follows that $N\left(M_{\lambda}\right) \subseteq M_{\lambda-1}$. In the following, we will use the notation:

$$
M_{\leq \lambda}:=\bigoplus_{\substack{\lambda^{\prime} \in \mathbb{Q} \\ \lambda^{\prime} \leq \lambda}} M_{\lambda^{\prime}}, \quad M_{\geq \lambda}:=\bigoplus_{\substack{\lambda^{\prime} \in \mathbb{Q} \\ \lambda^{\prime} \geq \lambda}} M_{\lambda^{\prime}} .
$$

Definition 1.1.3. We say that an object $(M, \phi, F) \in M F_{K}^{\phi}$ is a mixed Tate filtered $\phi$-module if the following properties are satisfied:

(1) There is an isomorphism of $\phi$-modules

$$
(M, \phi) \cong \bigoplus_{i \in I}\left(K_{0}, p^{n_{i}} \sigma\right)
$$

for some index set $I$, and $n_{i} \in \mathbb{Z}$.

(2) For all $i \in \mathbb{Z}$ the natural map

$$
F^{i} M_{K} \rightarrow M_{\geq i} \otimes_{K_{0}} K
$$

is an isomorphism.

We say that $(M, \phi, N, F) \in M F_{K}^{\phi, N}$ is a mixed Tate filtered $(\phi, N)$-module if $(M, \phi, F)$ is a mixed Tate filtered $\phi$-module.

We denote by $M T_{K}^{\phi}$ (resp. $\left.M T_{K}^{\phi, N}\right)$ the full subcategory of $M F_{K}^{\phi}\left(\right.$ resp. $M F_{K}^{\phi, N}$ ) with mixed Tate filtered $\phi$-modules (resp. $(\phi, N)$-modules) as objects. The categories $M T_{K}^{\phi}$ and $M T_{K}^{\phi, N}$ are additive. Again, we consider $M T_{K}^{\phi}$ as full subcategory of $M T_{K}^{\phi, N}$.

For $(M, \phi, N, F) \in M T_{K}^{\phi, N}$, it follows from Property (1) that all the slopes of $(M, \phi)$ are integers. From Property (2) we conclude that the Hodge polygon of $\left(M_{K}, F\right)$ equals the Newton polygon of $(M, \phi)$.

Definition 1.1.4 (Tate objects). Let $n \in \mathbb{Z}$ be an integer. We define the Tate object $K(n) \in M T_{K}^{\phi}$ by

$$
K(n):=\left(K_{0}, p^{-n} \sigma, F\right)
$$

with $F$ defined by

$$
F^{j}= \begin{cases}K, & \text { if } j \leq-n, \\ 0, & \text { if } j>-n .\end{cases}
$$

Definition 1.1.5 (Weight filtration). Let $(M, \phi, N, F) \in M T_{K}^{\phi, N}$. Let $i \in \mathbb{Z}$ be an integer. We define an object $W_{2 i}(M, \phi, N, F)$ in $M F_{K}^{\phi}$ by

$$
W_{2 i}(M, \phi, N, F):=\left(M_{\leq i},\left.\phi\right|_{M_{\leq i}},\left.N\right|_{M_{\leq i}}, F \cap M_{\leq i}\right) .
$$

We define an object $\operatorname{gr}_{2 i}^{W}(M, \phi, N, F)$ in $M T_{K}^{\phi}$ by

$$
\operatorname{gr}_{2 i}^{W}(M, \phi, N, F):=\left(M_{i},\left.\phi\right|_{M_{i}}, \tilde{F}\right),
$$


where $\tilde{F}$ is defined as follows:

$$
\tilde{F}^{i} M_{i}=M_{i}, \quad \tilde{F}^{i+1} M_{i}=0 .
$$

Note that $N\left(M_{\leq i}\right) \subset M_{\leq i-1}$ and $\left.N\right|_{M_{\leq i}}$ is well-defined.

Proposition 1.1.6. Let $(M, \phi, N, F) \in M T_{K}^{\phi, N}$ and $i \in \mathbb{Z}$. The following statements hold.

(1) The object $W_{2 i}(M, \phi, N, F)$ is contained in $M T_{K}^{\phi, N}$.

(2) There is an exact sequence

$$
0 \rightarrow W_{2(i-1)}(M, \phi, N, F) \rightarrow W_{2 i}(M, \phi, N, F) \rightarrow \operatorname{gr}_{2 i}^{W}(M, \phi, N, F) \rightarrow 0 .
$$

Proof. It is sufficient to prove the statement for $(M, \phi, 0, F)$, i.e., for objects in $M T_{K}^{\phi}$.

For (1). It is obvious that

$$
W_{2 i}\left(W_{2(i+1)}(M, \phi, F)\right)=W_{2 i}(M, \phi, F),
$$

for all $(M, \phi, F)$. Therefore, we may reduce to the case

$$
W_{2(i+1)}(M, \phi, F)=(M, \phi, F) .
$$

In this case $M=M_{\leq i} \oplus M_{i+1}$, and we have to prove that for all $j \in \mathbb{Z}$ the map

$$
F^{j} \cap\left(M_{\leq i} \otimes_{K_{0}} K\right) \rightarrow\left(M_{\leq i}\right)_{\geq j} \otimes_{K_{0}} K
$$

is an isomorphism. Since $(M, \phi, F)$ is an object in $M T_{K}^{\phi}$, the map is injective. In particular, the map is an isomorphism for all $j \geq i+1$.

We need to show the surjectivity for $j \leq i$. By assumption, for every $m \in$ $\left(M_{\leq i}\right)_{\geq j} \otimes_{K_{0}} K$ there exists a preimage $m^{\prime} \in F^{j} M_{K}$. By definition, the projection of $m^{\prime}$ to $M_{i+1} \otimes_{K_{0}} K$ vanishes, thus $m^{\prime} \in F^{j} \cap\left(M_{\leq i} \otimes_{K_{0}} K\right)$.

For (2). There is an obvious morphism $W_{2(i-1)}(M, \phi, F) \rightarrow W_{2 i}(M, \phi, F)$ in $M T_{K}^{\phi}$. The morphism $W_{2 i}(M, \phi, F) \rightarrow \operatorname{gr}_{2 i}^{W}(M, \phi, F)$ is defined by the projection $M_{\leq i} \rightarrow M_{i}$. Since $F^{i+1} \cap\left(M_{\leq i} \otimes_{K_{0}} K\right)=0$, the projection is compatible with the filtrations. Therefore the sequence (1.1.1) is well-defined.

In order to prove that the sequence is exact we need to show that it is an exact sequence of $\phi$-modules and an exact sequence of filtered $K$-vector spaces. The first statement is obvious. For the second statement we note that all members in the sequence (1.1.1) are objects in $M T_{K}^{\phi}$, thus the Hodge polygons equal the Newton polygons. In particular,

$$
\operatorname{dim}\left(F^{j} \cap M_{\leq i}\right)=\operatorname{dim}\left(F^{j} \cap M_{\leq i-1}\right)+\operatorname{dim} \tilde{F}^{j},
$$

for all $j \in \mathbb{Z}$. This immediately implies the claim.

Corollary 1.1.7. The category $M T_{K}^{\phi, N}$ is contained in the category of weakly admissible filtered $(\phi, N)$-modules.

Proof. We use the fact that weakly admissible filtered $(\phi, N)$-modules are stable under extensions. Therefore the claim follows from Proposition 1.1.6 provided we prove that $\operatorname{gr}_{2 i}^{W}(M, \phi, N, F)$ is weakly admissible for all $(M, \phi, N, F) \in M T_{K}^{\phi, N}$ and all $i \in \mathbb{Z}$. By Definition 1.1.5, $\operatorname{gr}_{2 i}^{W}(M, \phi, N, F)$ is isomorphic to a direct sum of Tate objects $K(-i)$. Since Tate objects are (weakly) admissible, we are done. 
In contrast to the category $M F_{K}^{\phi, N}$, the category of weakly admissible filtered $(\phi, N)$-modules $M F_{K}^{\phi, N, w a}$ is an abelian category.

Proposition 1.1.8. Let $f:\left(M, \phi_{M}, N_{M}, F_{M}\right) \rightarrow\left(M^{\prime}, \phi_{M^{\prime}}, N_{M^{\prime}}, F_{M^{\prime}}\right)$ be a morphism in $M T_{K}^{\phi, N}$. We denote by $\operatorname{ker}(f)$ and coker $(f)$ the kernel of $f$ and the cokernel of $f$ in $M F_{K}^{\phi, N, w a}$, respectively. Then $\operatorname{ker}(f)$ and coker $(f)$ are contained in $M T_{K}^{\phi, N}$. In particular, $M T_{K}^{\phi, N}$ is an abelian category.

Proof. First, consider the full subcategory $\mathcal{C}$ of isocrystals over $K_{0}$ with objects $(M, \phi)$ such that there exists an isomorphism

$$
(M, \phi) \cong \bigoplus_{i \in I}\left(K_{0}, p^{n_{i}} \sigma\right) .
$$

It is easy to see that $\mathcal{C}$, as subcategory of the category of isocrystals, contains all the kernels and cokernels of morphisms in $\mathcal{C}$.

We denote by $f_{0}$ the induced morphism $\left(M, \phi_{M}\right) \rightarrow\left(M^{\prime}, \phi_{M^{\prime}}\right)$. Then

$$
\operatorname{ker}(f)=\left(\operatorname{ker}\left(f_{0}\right),\left.\phi\right|_{\operatorname{ker}\left(f_{0}\right)},\left.N\right|_{\operatorname{ker}\left(f_{0}\right)}, F \cap\left(\operatorname{ker}\left(f_{0}\right) \otimes_{K_{0}} K\right)\right) .
$$

We know that $\operatorname{ker}\left(f_{0}\right) \in \mathcal{C}$ and thus satisfies Property (1) of Definition 1.1.3. It remains to show that

$$
F_{M}^{i} \cap\left(\operatorname{ker}\left(f_{0}\right) \otimes_{K_{0}} K\right) \rightarrow \operatorname{ker}\left(f_{0}\right)_{\geq i} \otimes_{K_{0}} K
$$

is an isomorphism. We have a commutative diagram

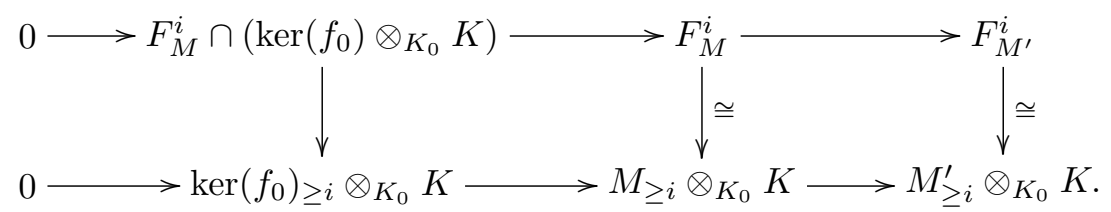

Moreover, both rows are exact, which implies Property (2) of Definition 1.1.3.

The claim for the cokernel follows dually.

1.1.9. The categories $M T_{K}^{\phi, N}$ and $M T_{K}^{\phi}$ are $\mathbb{Q}_{p}$-linear rigid $\otimes$-categories.

Lemma 1.1.10. The functor

$$
\tilde{\omega}: M T_{K}^{\phi, N} \rightarrow\left(\mathbb{Q}_{p} \text {-vector spaces }\right), \quad(M, \phi, N, F) \mapsto \bigoplus_{n \in \mathbb{Z}} \tilde{\omega}_{n}(M, \phi, F),
$$

with

$$
\tilde{\omega}_{n}(M, \phi, F)=\operatorname{Hom}_{M T_{K}^{\phi}}\left(K(n), \operatorname{gr}_{-2 n}^{W}(M, \phi, F)\right),
$$

is a fibre functor. In particular, $\left(M T_{K}^{\phi, N}, \tilde{\omega}\right)$ and $\left(M T_{K}^{\phi}, \tilde{\omega}\right)$ are Tannaka categories.

Proof. It is easy to see that $\tilde{\omega}$ is a $\otimes$-functor. In order to see that $\tilde{\omega}$ is exact and faithful we will prove the existence of an isomorphism

$$
\tilde{\omega}_{K_{0}} \stackrel{\cong}{\longrightarrow}(\gamma:(M, \phi, N, F) \mapsto M),
$$

where $\tilde{\omega}_{K_{0}}(M, \phi, N, F)=\tilde{\omega}(M, \phi, N, F) \otimes_{\mathbb{Q}_{p}} K_{0}$ and $\gamma$ forgets about $\phi, N$ and $F$. Since $\gamma$ is exact and faithful, this will imply the claim. 
In order to construct (1.1.3), we observe that there is a functorial isomorphism

$$
\begin{gathered}
\operatorname{Hom}_{M T_{K}^{\phi}}\left(K(n), \operatorname{gr}_{-2 n}^{W}(M, \phi, N, F)\right) \otimes_{\mathbb{Q}_{p}} K_{0} \rightarrow M_{-n}, \\
\phi \otimes a \mapsto a \cdot \phi(1) .
\end{gathered}
$$

Proposition 1.1.11. An object $(M, \phi, N, F) \in M F_{K}^{\phi, N, w a}$ belongs to $M T_{K}^{\phi, N}$ if and only if there exists an increasing exhaustive separated filtration $W$ by subobjects of $(M, \phi, N, F)$ in $M F_{K}^{\phi, N, w a}$ such that $W_{i} / W_{i-1}$ vanishes if $i$ is odd, and is a sum of Tate objects $K\left(-\frac{i}{2}\right)$ if $i$ is even.

Proof. For $(M, \phi, N, F) \in M T_{K}^{\phi, N}$, such a filtration exists by Definition 1.1.5, Proposition 1.1.6, and the fact that $\operatorname{gr}_{2 i}^{W}(M, \phi, N, F)$ is a sum of Tate objects $K(-i)$.

Suppose now that $(M, \phi, N, F) \in M F_{K}^{\phi, N, w a}$ admits a filtration $W$ satisfying the assumptions. It is easy to see that $(M, \phi)$ satisfies Property (1) of Definition 1.1.3.

In general, if

$$
0 \rightarrow M_{1} \rightarrow M \rightarrow M_{2} \rightarrow 0
$$

is an exact sequence in $M F_{K}^{\phi, N, w a}$, and $M_{1}, M_{2}$ satisfy Property (2), then $M$ satisfies Property (2). By induction on $i$ we conclude that $W_{i} \in M T_{K}^{\phi, N}$ for all $i$.

It is clear that any filtration as in Proposition 1.1.11 has to coincide with the weight filtration, and that any morphism between two objects in $M T_{K}^{\phi, N}$ has to be strict with respect to the weight filtrations on these objects.

\subsection{The crystalline logarithmic point.}

1.2.1. Recall from (1.1.2) that we have a fibre functor $\tilde{\omega}$ equipping $M T_{K}^{\phi}$ and $M T_{K}^{\phi, N}$ with the structure of Tannaka categories (Lemma 1.1.10). Let $G_{\tilde{\omega}}$ and $G_{\tilde{\omega}}^{s t}$ denote the pro-algebraic groups which represent tensor automorphisms of $\tilde{\omega}$ on $M T_{K}^{\phi}$ and $M T_{K}^{\phi, N}$, respectively. In other words, we have $G_{\tilde{\omega}}=\underline{\mathrm{Aut}}_{M T_{K}^{\phi}}^{\otimes} \tilde{\omega}$ and $G_{\tilde{\omega}}^{s t}=\underline{\mathrm{Aut}}_{M T_{K}^{\phi, N}}^{\otimes} \tilde{\omega}$. The goal of this section is to construct a non-trivial $K$-valued point $\eta$ of $G_{\tilde{\omega}}$.

Definition 1.2.2. For $(M, \phi, F) \in M T_{K}^{\phi}$ we define

$$
\eta(M, \phi, F): M_{K} \rightarrow M_{K}
$$

to be the unique endomorphism rendering the following diagram commutative:

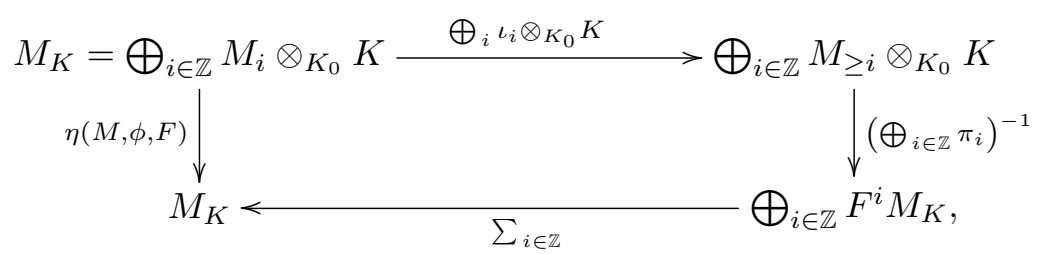

where $\iota_{i}: M_{i} \rightarrow M_{\geq i}$ is the obvious inclusion, $\pi_{i}: F^{i} M_{K} \rightarrow M_{\geq i} \otimes_{K_{0}} K$ is the projection and therefore by definition an isomorphism (Definition 1.1.3(2)), and $\sum_{i \in \mathbb{Z}}$ is the sum over the obvious inclusions.

Lemma 1.2.3. The morphisms $\eta$ from Definition 1.2.2 define a tensor automorphism of the fibre functor $\tilde{\omega}_{K}=\tilde{\omega} \otimes_{\mathbb{Q}_{p}} K$. 
Proof. Via the $\otimes$-isomorphism (1.1.3) we may identify $\tilde{\omega} \otimes_{\mathbb{Q}_{p}} K_{0}$ with the forgetful functor $(M, \phi, F) \mapsto M$. After tensoring with $K$ we obtain $\tilde{\omega}_{K}(M, \phi, F)=M_{K}$.

First, let us prove that $\eta(M, \phi, F)$ is an automorphism. We denote by

$$
\eta(M, \phi, F)[i, j]: M_{j} \otimes_{K_{0}} K \rightarrow M_{i} \otimes_{K_{0}} K
$$

the composition with the inclusion $M_{j} \otimes_{K_{0}} K \rightarrow M_{K}$ and the projection $M_{K} \rightarrow$ $M_{i} \otimes_{K_{0}} K$. The following claim implies that $\eta(M, \phi, F)$ is an automorphism.

Claim 1.2.4.

$$
\eta(M, \phi, F)[i, j]= \begin{cases}0, & \text { if } i>j, \\ i d_{M_{i} \otimes_{K_{0}} K}, & \text { if } i=j .\end{cases}
$$

Proof of Claim 1.2.4. For $a \in M_{j} \otimes_{K_{0}} K$ we have $\eta(M, \phi, F)(a)=\pi_{j}^{-1}(a)$, where $\pi_{j}: F^{j} M_{K} \rightarrow M_{\geq j} \otimes_{K_{0}} K$ is the projection. For $i \geq j$ the projection $M_{K} \rightarrow M_{i} \otimes_{K_{0}} K$ factors as

$$
M_{K} \stackrel{\pi_{j}}{\longrightarrow} M_{\geq j} \otimes_{K_{0}} K=\bigoplus_{l \geq j} M_{l} \otimes_{K_{0}} K \stackrel{\text { projection }}{\longrightarrow} M_{i} \otimes_{K_{0}} K .
$$

Since $\pi_{j} \circ \eta(M, \phi, F)(a)=a$ is concentrated in the $j$-th component, the claim follows.

Since the diagram (1.2.1) is functorial, $\eta$ defines a natural transformation. The compatibility with the tensor product is obvious.

1.2.5. By Lemma 1.2 .3 , we obtain a $K$-valued point $\eta \in G_{\tilde{\omega}}(K)$; we call this point the logarithmic point. Let us check that $\eta$ is not the identity.

Proposition 1.2.6. Let $n \in \mathbb{Z}$ be an integer. We have

$$
\operatorname{Ext}_{M T_{K}^{\phi}}^{1}(K(0), K(n)) \cong \begin{cases}K, & \text { if } n>0 \\ 0, & \text { if } n \leq 0\end{cases}
$$

Let

$$
0 \rightarrow K(n) \stackrel{\iota}{\rightarrow}(E, \phi, F) \stackrel{\pi}{\rightarrow} K(0) \rightarrow 0
$$

be an extension. For $n \neq 0$, there are unique sections $f: E \rightarrow K_{0}$ and $v: K_{0} \rightarrow E$ of the underlying maps of $K_{0}$-isocrystals of $\iota$ and $\pi$, respectively. The isomorphism (1.2.3), for $n \neq 0$, is given by the formula

$$
E \mapsto f(\eta(E, \phi, F)(v(1))) .
$$

Proof. First, we consider the case $n=0$. Let $(E, \phi, F)$ be as in (1.2.4). We have $F^{0}\left(E_{K}\right)=E_{K}$ and $F^{1}\left(E_{K}\right)=0$ by Definition 1.1.3(2). In view of Definition 1.1.3(2) there is an isomorphism $(E, \phi) \cong\left(K_{0}, \sigma\right) \oplus\left(K_{0}, \sigma\right)$, thus there is a section of $\pi$ in $M T_{K}^{\phi}$.

For $n \neq 0$ : From the slope decomposition we obtain natural sections $f, v$ as $\phi$-modules. If $n<0$ then $F^{1} E_{K}=\iota(K)$ which means $(E, \phi, F)=K(0) \oplus K(n)$.

For $n>0$, we can uniquely write $F^{-n+1} E_{K}=K\langle a \cdot \iota(1)+v(1)\rangle$ with $a \in K$. Let us compute $f(\eta(E, \phi, F)(v(1)))$. Since $v(1) \in E_{0}$, we have $\eta(E, \phi, F)(v(1))=$ $\pi_{0}^{-1}(v(1))$. On the other hand, $a \cdot \iota(1)+v(1) \in F^{-n+1} E_{K}=F^{0} E_{K}$, and maps to $v(1)$ under $\pi_{0}$. Therefore $\eta(E, \phi, F)(v(1))=\pi_{0}^{-1}(v(1))=a \cdot \iota(1)+v(1)$ and 
hence $f(\eta(E, \phi, F)(v(1)))=a$. It is clear that $F^{-n+1} E_{K}$ is the only invariant for extensions.

1.2.7. Recall that we have a fibre functor $\tilde{\omega}(1.1 .2)$ to the category of $\mathbb{Q}_{p}$-vector spaces. In the obvious way $\tilde{\omega}$ factors through the category of graded $\mathbb{Q}_{p}$-vector spaces. Furthermore, we have an automorphism $\eta$ of $\tilde{\omega}_{K}$ (Lemma 1.2.3).

Definition 1.2.8. We define $\mathcal{C}_{\eta}$ to be the category of pairs $(V, \eta)$, where $V$ is a finitedimensional graded $\mathbb{Q}_{p}$-vector space and $\eta: V \otimes K \rightarrow V \otimes K$ is a $K$-linear map such that for all $n \in \mathbb{Z}$ :

$$
(\eta-i d)\left(V_{n} \otimes K\right) \subset \bigoplus_{i>n} V_{i} \otimes K
$$

Morphisms $\left(V_{1}, \eta_{1}\right) \rightarrow\left(V_{2}, \eta_{2}\right)$ are $\mathbb{Q}_{p}$-linear morphisms $\tau: V_{1} \rightarrow V_{2}$ which respect the grading and commute with the endomorphisms $\eta_{i}$, i.e., $\eta_{2} \circ\left(\tau \otimes i d_{K}\right)=\left(\tau \otimes i d_{K}\right) \circ \eta_{1}$.

The category $\mathcal{C}_{\eta}$ is a $\otimes$-category with

$$
\left(V_{1}, \eta_{1}\right) \otimes\left(V_{2}, \eta_{2}\right)=\left(V_{1} \otimes V_{2}, \eta_{1} \otimes \eta_{2}\right) .
$$

Proposition 1.2.9. The functor

$$
\begin{aligned}
\Psi: M T_{K}^{\phi} & \rightarrow \mathcal{C}_{\eta} \\
(M, \phi, F) & \mapsto\left(\bigoplus_{n \in \mathbb{Z}} \tilde{\omega}_{n}(M, \phi, F), \eta(M, \phi, F)\right)
\end{aligned}
$$

is an equivalence of $\otimes$-categories.

Proof. By Lemma 1.2.3, $\eta$ is functorial and $\Psi$ is a $\otimes$-functor. It follows from (1.2.2) that

$$
(\eta-i d)\left(\tilde{\omega}_{n} \otimes K\right) \subset \bigoplus_{i>n} \tilde{\omega}_{i} \otimes K
$$

We define a functor

$$
\begin{aligned}
\Phi: \mathcal{C}_{\eta} & \rightarrow M T_{K}^{\phi} \\
\left(\oplus_{n \in \mathbb{Z}} V_{n}, \eta\right) & \mapsto\left(\oplus_{n \in \mathbb{Z}}\left(V_{-n} \otimes_{\mathbb{Q}_{p}} K_{0}, p^{-n} \otimes \sigma\right), F\right),
\end{aligned}
$$

with the following filtration:

$$
F^{i}:=\eta\left(\bigoplus_{j \geq i} V_{-j} \otimes_{\mathbb{Q}_{p}} K\right),
$$

for all $i$. Property (1.2.5) implies that $\Phi$ is well-defined. From Definition 1.2.2 it easily follows that $\Psi \circ \Phi=i d_{\mathcal{C}_{\eta}}$.

On the other hand, we have $\Phi \circ \Psi \stackrel{\cong}{\longrightarrow} i d_{M T_{K}^{\phi}}$ via

$$
\begin{array}{r}
\Phi \circ \Psi(M, \phi, F) \rightarrow(M, \phi, F) \\
\bigoplus_{n \in \mathbb{Z}} \tilde{\omega}_{-n}(M, \phi, F) \otimes_{\mathbb{Q}_{p}} K_{0} \stackrel{(1.1 .4)}{\longrightarrow} M .
\end{array}
$$




\subsection{The semistable logarithmic point.}

1.3.1. Let $K$ be as in Section 1.1.1 with residue field $k$. We denote by $\nu_{K}$ the valuation of $K$.

1.3.2. Recall that we have a homomorphism

$$
[.]: k^{\times} \rightarrow \mathcal{O}_{K}^{\times}, \quad x \mapsto[x],
$$

by taking the Teichmüller lift. Denoting by $U_{K}:=\left\{x \in \mathcal{O}_{K}^{\times} ; x \in 1+m_{K}\right\}$ the 1-units, we obtain a decomposition

$$
\mathcal{O}_{K}^{\times}=k^{\times} \times U_{K}
$$

The logarithm

$$
\log : \mathcal{O}_{K}^{\times} \rightarrow \mathcal{O}_{K}
$$

is by definition trivial on the factor $k^{\times}$and is given by

$$
\log (u)=\sum_{n \geq 1}(-1)^{n+1} \frac{(u-1)^{n}}{n}, \quad \text { for all } u \in U_{K} .
$$

1.3.3. We consider $\mathcal{O}_{K, \mathbb{Q}}^{\times}:=\mathcal{O}_{K}^{\times} \otimes_{\mathbb{Z}} \mathbb{Q}$ and $K_{\mathbb{Q}}^{\times}:=K^{\times} \otimes_{\mathbb{Z}} \mathbb{Q}$ as $\mathbb{Q}$-vector spaces, therefore we may form the symmetric algebras $\operatorname{Sym}_{\mathbb{Q}}\left(\mathcal{O}_{K, \mathbb{Q}}^{\times}\right)$and $\operatorname{Sym}_{\mathbb{Q}}\left(K_{\mathbb{Q}}^{\times}\right)$. The exact sequence

$$
0 \rightarrow \mathcal{O}_{K, \mathbb{Q}}^{\times} \rightarrow K_{\mathbb{Q}}^{\times} \stackrel{\nu_{K}}{\longrightarrow} \mathbb{Q} \rightarrow 0
$$

implies that $\operatorname{Spec}\left(\operatorname{Sym}_{\mathbb{Q}}\left(K_{\mathbb{Q}}^{\times}\right)\right)$is a one-dimensional affine space over Spec $\left(\operatorname{Sym}_{\mathbb{Q}}\left(\mathcal{O}_{K, \mathbb{Q}}^{\times}\right)\right)$. In other words, for $x \in K^{\times}$with $\nu_{K}(x) \neq 0$, the map

$$
\operatorname{Sym}_{\mathbb{Q}}\left(\mathcal{O}_{K, \mathbb{Q}}^{\times}\right)[X] \rightarrow \operatorname{Sym}_{\mathbb{Q}}\left(K_{\mathbb{Q}}^{\times}\right), \quad X \mapsto x,
$$

is an isomorphism.

The logarithm (1.3.1) induces a ring homomorphism

$$
\operatorname{Sym}_{\mathbb{Q}}\left(\mathcal{O}_{K, \mathbb{Q}}^{\times}\right) \rightarrow K \text {. }
$$

Definition 1.3.4. We define the $K$-algebra $K_{s t}$ by

$$
K_{s t}:=\operatorname{Sym}_{\mathbb{Q}}\left(K_{\mathbb{Q}}^{\times}\right) \otimes_{\operatorname{Sym}_{\mathbb{Q}}\left(\mathcal{O}_{K, \mathbb{Q}}^{\times}\right)} K .
$$

By base change, $\operatorname{Spec}\left(K_{s t}\right)$ is a one-dimensional affine space over $K$. We have a natural logarithm

$$
\log _{s t}: K^{\times} \rightarrow K_{s t}, \quad x \mapsto x \otimes 1 .
$$

The $K$-valued points of $\operatorname{Spec}\left(K_{s t}\right)$ admit the following description:

$$
\begin{aligned}
\operatorname{Spec}\left(K_{s t}\right)(K) & =\left\{\text { extensions } \log : K^{\times} \rightarrow K \text { of }(1.3 .1)\right\} \\
f & \mapsto f^{*} \circ \log _{s t} .
\end{aligned}
$$

By an extension log : $K^{\times} \rightarrow K$ we mean a homomorphism such that the restriction to $\mathcal{O}_{K}^{\times}$equals $(1.3 .1)$. 
1.3.5. The $p$-adic Hodge theory of $K$ (and fixed valuation $\nu_{K}$ ) depends for semistable representations on the choice of a logarithm

$$
\log : K^{\times} \rightarrow K
$$

It will be important for us that our constructions do not depend on a particular choice, and for this we have to recall the basic constructions of $p$-adic Hodge theory.

We denote by $R$ the ring

$$
R:=\lim _{\longleftarrow} \mathcal{O}_{\bar{K}} / p \mathcal{O}_{\bar{K}},
$$

where the maps are given by rising to the $p$-th power $x \mapsto x^{p}$. Denoting by $C_{K}=\widehat{\bar{K}}$ the $p$-adic completion of $\bar{K}$ we have a multiplicative bijection

$$
\stackrel{\lim }{\longleftarrow} \mathcal{O}_{C_{K}} \rightarrow R
$$

where the projective system is defined by rising to the $p$-th power again. In other words, we can represent every element $x$ in $R$ by $\left(x^{(0)}, x^{(1)}, \ldots\right)$ with $x^{(n)} \in \mathcal{O}_{C_{K}}$ and $x^{(n-1)}=\left(x^{(n)}\right)^{p}$.

Let $\nu_{\bar{K}}\left(\right.$ resp. $\left.\nu_{C_{K}}\right)$ be the extension of $\nu_{K}\left(\right.$ resp. $\left.\nu_{\bar{K}}\right)$ to $\bar{K}\left(\operatorname{resp} . C_{K}\right)$. The map

$$
\nu_{R}: R \backslash\{0\} \rightarrow \mathbb{Q}, \quad x \mapsto \nu_{C_{K}}\left(x^{(0)}\right)
$$

can be extended to a valuation

$$
\nu_{R}: \operatorname{Frac}(R)^{\times} \rightarrow \mathbb{Q}
$$

with valuation $\operatorname{ring} R$.

Let $B_{\text {cris }}$ be the crystalline period ring; we define

$$
B_{\text {st }}=\operatorname{Sym}_{\mathbb{Q}}\left(\operatorname{Frac}(R)^{\times} \otimes_{\mathbb{Z}} \mathbb{Q}\right) \otimes_{\operatorname{Sym}_{\mathbb{Q}}\left(R^{\times} \otimes_{\mathbb{Z}} \mathbb{Q}\right)} B_{\text {cris }},
$$

where $\operatorname{Sym}_{\mathbb{Q}}\left(R^{\times} \otimes_{\mathbb{Z}} \mathbb{Q}\right) \rightarrow B_{\text {cris }}$ is induced by the crystalline logarithm

$$
\log _{\text {cris }}: R^{\times} \rightarrow B_{\text {cris }} .
$$

Again, $R^{\times}=\bar{k}^{\times} \times\left(1+m_{R}\right)$; $\log _{\text {cris }}$ is trivial on $\bar{k}^{\times}$and given by

$$
\log _{\text {cris }}(u)=\sum_{n \geq 1}(-1)^{n+1} \frac{([u]-1)^{n}}{n}
$$

for $u \in 1+m_{R}$, where $[u]$ denotes the Teichmüller lift of $u$ in the Witt $\operatorname{ring} W(R)$ of $R$.

By construction we have a natural logarithm

$$
\log _{s t}: \operatorname{Frac}(R)^{\times} \rightarrow B_{s t}, \quad x \mapsto x \otimes 1 .
$$

The ring $B_{s t}$ has the following properties.

(1) We have a $\operatorname{Gal}(\bar{K} / K)$-action on $B_{\text {st }}$ extending the action on $B_{\text {cris }}$.

(2) We have a Frobenius map $\phi: B_{s t} \rightarrow B_{s t}$ extending the Frobenius map on $B_{\text {cris. }}$ Moreover,

$$
\phi \circ \log _{s t}=p \log _{s t} .
$$

(3) We have a $B_{\text {cris }}$-linear derivation $N: B_{s t} \rightarrow B_{s t}$ such that

$$
N\left(\log _{s t}(x)\right)=\nu_{R}(x), \quad \text { for all } x \in \operatorname{Frac}(R)^{\times} .
$$


After choosing a logarithm

$$
\log : K^{\times} \rightarrow K
$$

which extends (1.3.1), we obtain a morphism of $B_{\text {cris-algebras }}$

$$
\gamma_{\log }: B_{s t} \rightarrow B_{d R}
$$

We denote by $\gamma_{\text {log, } K}: B_{s t} \otimes_{K_{0}} K \rightarrow B_{d R}$ the induced map of $B_{\text {cris }} \otimes_{K_{0}} K$-algebras. The morphism depends on the choice of log, and the filtration on $B_{s t} \otimes_{K_{0}} K$ induced by the filtration on $B_{d R}$ via $\gamma_{\log , K}$ depends on $\log$.

Proposition 1.3.6. For $\log , \log ^{\prime} \in \operatorname{Spec}\left(K_{s t}\right)(K)$ there is a unique ring homomorphism

$$
\delta_{\log , \log ^{\prime}}: B_{s t} \otimes_{K_{0}} K \rightarrow B_{s t} \otimes_{K_{0}} K
$$

such that $\gamma_{\log ^{\prime}, K} \circ \delta_{\log , \log ^{\prime}}=\gamma_{\log , K}$. The map $\delta_{\log , \log ^{\prime}}$ is given by

$$
\delta_{\log , \log ^{\prime}}=\exp \left(\frac{\log (x)-\log ^{\prime}(x)}{\nu_{K}(x)} N\right)
$$

for every $x \in K \backslash \mathcal{O}_{K}^{\times}$.

Proof. Uniqueness follows from the fact that $\gamma_{\mathrm{log}, K}$ is injective.

Choose $\tilde{p} \in R$ with $\tilde{p}^{(0)}=p$. By definition we have

$$
\gamma_{\log }\left(\log _{s t}(\tilde{p})\right)=\log _{d R}([\tilde{p}] / p)+\log (p),
$$

where $\log _{d R}$ is defined by the usual series since $[\tilde{p}] / p$ is a 1-unit in $B_{d R}$. Since $\operatorname{Spec}\left(B_{s t}\right)$ is a one-dimensional affine space over $\operatorname{Spec}\left(B_{\text {cris }}\right)$, there exists a unique morphism of $B_{\text {cris }} \otimes_{K_{0}} K$-algebras $\delta_{\text {log,log' }}$ such that

$$
\delta_{\log , \log ^{\prime}}\left(\log _{s t}(\tilde{p})\right)=\log _{s t}(\tilde{p})+\log (p)-\log ^{\prime}(p) .
$$

Obviously, $\delta_{\log , \log ^{\prime}}$ satisfies $\gamma_{\log ^{\prime}, K} \circ \delta_{\log , \log ^{\prime}}=\gamma_{\log , K}$ and the equality (1.3.5).

By using $\gamma_{\log , K}$ we obtain a filtration on $B_{s t} \otimes_{K_{0}} K$. The $p$-adic Hodge theory [2, Thm. A] asserts that the functor

$$
\begin{gathered}
D_{s t, \log }:\left(\text { semistable } \mathbb{Q}_{p} \text {-representations of } \operatorname{Gal}(\bar{K} / K)\right) \rightarrow M F_{K}^{\phi, N, w \cdot a .} \\
V \mapsto\left(B_{s t} \otimes_{\mathbb{Q}_{p}} V\right)^{\operatorname{Gal}(\bar{K} / K)}
\end{gathered}
$$

is an equivalence of categories. We will use the subscript $\log$ in $D_{s t, \log }$ to emphasize the dependence on $\log$.

Denoting by forget $_{F}$ the functor forget $_{F}(M, \phi, N, F)=(M, \phi, N)$, we get

$$
\text { forget }_{F} \circ D_{s t, \log }=\text { forget }_{F} \circ D_{s t, \log ^{\prime}},
$$

because only the filtration depends on the embedding to $B_{d R}$. Proposition 1.3.6 implies that for the filtrations we have the following comparison:

$$
F_{D_{s t, \log ^{\prime}}(V)}^{i}=\exp \left(\frac{\log (x)-\log ^{\prime}(x)}{\nu_{K}(x)} N\right) F_{D_{s t, \log }(V)}^{i},
$$

for all $i \in \mathbb{Z}$ and all $x \in K \backslash \mathcal{O}_{K}^{\times}$. 
Definition 1.3.7. Let $K$ be a $p$-adic field. We denote by $M T_{G_{K}}$ the full subcategory of $p$-adic representations $V$ of $\operatorname{Gal}(\bar{K} / K)$ which admit an increasing exhaustive separated filtration $W$ by subrepresentations of $V$ such that $W_{i} / W_{i-1}$ vanishes if $i$ is odd, and is a sum of Tate objects $\mathbb{Q}_{p}\left(-\frac{i}{2}\right)$ if $i$ is even. We call an object of $M T_{G_{K}}$ a mixed Tate representation of $\operatorname{Gal}(\bar{K} / K)$.

Proposition 1.3.8. Let $\log \in \operatorname{Spec}\left(K_{s t}\right)(K)$. Then

$$
M T_{G_{K}}=D_{s t, \log }^{-1}\left(M T_{K}^{\phi, N}\right) .
$$

In particular, every mixed Tate representation is semistable.

Proof. From Proposition 1.1.11 it follows that every object in $D_{s t, \log }^{-1}\left(M T_{K}^{\phi, N}\right)$ admits a filtration $W$ satisfying the properties of Definition 1.3.7.

Now, suppose that $V$ is a $p$-adic representation of $\operatorname{Gal}(\bar{K} / K)$ which admits a filtration $W$ as in Definition 1.3.7. If we know that $V$ is semistable then clearly $D_{s t, \log }(V) \in M T_{K}^{\phi, N}$ by Proposition 1.1.11, again. Therefore it suffices to prove that $V$ is semistable.

We use induction on the length of the filtration $W$ of $V$. If the filtration $W$ has length $\leq 1$, semistability of $V$ follows from those of $\mathbb{Q}_{p}(n)$. In general, let $n$ be the smallest integer such that $W_{2 n} V=V$. Then we have an exact sequence

$$
0 \rightarrow\left(W_{2 n-2} V\right) \otimes \mathbb{Q}_{p}(n) \rightarrow V \otimes \mathbb{Q}_{p}(n) \rightarrow\left(V / W_{2 n-2} V\right) \otimes \mathbb{Q}_{p}(n) \rightarrow 0 .
$$

By the induction hypothesis the terms on the left and right are semistable. Moreover, since the weights of the term on the left are $\leq-2$ and the term on the right has weight 0 , we have

$$
\begin{aligned}
F^{0} D_{d R}\left(\left(W_{2 n-2} V\right) \otimes \mathbb{Q}_{p}(n)\right) & =0 \\
F^{0}\left(D_{d R}\left(\left(V / W_{2 n-2} V\right) \otimes \mathbb{Q}_{p}(n)\right)\right) & =D_{d R}\left(\left(V / W_{2 n-2} V\right) \otimes \mathbb{Q}_{p}(n)\right) .
\end{aligned}
$$

Therefore [5, Proposition 1.28] shows that the middle term is also semistable.

Obviously,

$$
\tau=\tilde{\omega} \circ D_{s t, \log }
$$

is independent of $\log$, and $\left(M T_{G_{K}}, \tau\right)$ is a Tannaka category (by Lemma 1.1.10).

1.3.9. Recall from Lemma 1.1 .10 that $\left(M T_{K}^{\phi, N}, \tilde{\omega}\right)$ is a Tannaka category. We will use the ring $K_{s t}$ (Definition 1.3.4) and $\log _{s t}(1.3 .3)$.

Definition 1.3.10. For a logarithm $\log \in \operatorname{Spec}\left(K_{s t}\right)(K)$ and $(M, \phi, N, F) \in M T_{K}^{\phi, N}$ we define $\eta_{s t, \log }(M, \phi, N, F) \in \operatorname{End}_{K_{s t}}\left(M \otimes_{K_{0}} K_{s t}\right)$ by

$$
\eta_{s t, \log }(M, \phi, N, F):=\exp \left(\frac{\log (x)-\log _{s t}(x)}{\nu_{K}(x)} N\right) \eta(M, \phi, F),
$$

for $x \in K^{\times} \backslash \mathcal{O}_{K}^{\times}$. For the definition of $\eta(M, \phi, F)$ we refer to Definition 1.2.2.

Obviously, $\eta_{s t, \log }$ does not depend on the choice $x \in K^{\times} \backslash \mathcal{O}_{K}^{\times}$, but it depends on log. 


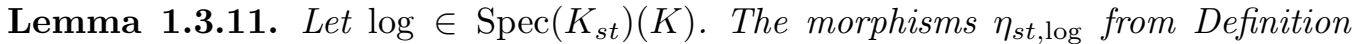
1.3 .10 define a tensor automorphism of the fibre functor $\tilde{\omega}_{K_{s t}}=\tilde{\omega} \otimes_{\mathbb{Q}_{p}} K_{\text {st }}$. In other words, $\eta_{s t, \log } \in G_{\tilde{\omega}}^{s t}\left(K_{s t}\right)$ with $G_{\tilde{\omega}}^{s t}=\underline{\operatorname{Aut}}_{M T_{K}^{\phi, N}}^{\otimes} \tilde{\omega}$.

Proof. Via the $\otimes$-isomorphism (1.1.3) we may identify $\tilde{\omega} \otimes_{\mathbb{Q}_{p}} K_{0}$ with the forgetful functor $(M, \phi, N, F) \mapsto M$. After tensoring with $K_{s t}$ we obtain $\tilde{\omega}_{K_{s t}}(M, \phi, N, F)=$ $M \otimes_{K_{0}} K_{s t}$. Lemma 1.2.3 implies that $\eta(M, \phi, F)$ is a tensor automorphism, thus it suffices to prove the statement for $\exp \left(\frac{\log (x)-\log _{s t}(x)}{\nu_{K}(x)} N\right)$. The functoriality follows immediately. The compatibility with the $\otimes$-structure follows from

$$
N_{M_{1} \otimes M_{2}}=N_{M_{1}} \otimes 1+1 \otimes N_{M_{2}} .
$$

Lemma 1.3.12. The $K_{\text {st }}$-valued point

$$
\eta_{s t}=\eta_{s t, \log } \circ D_{s t, \log }
$$

of $\underline{\mathrm{Aut}}_{M T_{G_{K}}}^{\otimes} \tau$ is independent of the choice of $\log \in \operatorname{Spec}\left(K_{s t}\right)(K)$.

Proof. Let $\log , \log ^{\prime} \in \operatorname{Spec}\left(K_{s t}\right)(K)$ and $V \in M T_{G_{K}}$. In view of (1.3.7) we get

$$
\eta\left(\text { forget }_{N} D_{s t, \log ^{\prime}}(V)\right)=\exp \left(\frac{\log (x)-\log ^{\prime}(x)}{\nu_{K}(x)} N\right) \eta\left(\operatorname{forget}_{N} D_{s t, \log }(V)\right),
$$

for very $x \in K \backslash \mathcal{O}_{K}^{\times}$, and forget $_{N}(M, \phi, N, F)=(M, \phi, F)$. Thus,

$$
\begin{aligned}
\eta_{s t, \log ^{\prime}} D_{s t, \log ^{\prime}}(V) & =\exp \left(\frac{\log ^{\prime}(x)-\log _{s t}(x)}{\nu_{K}(x)} N\right) \eta\left(\text { forget }_{N} D_{s t, \log ^{\prime}}(V)\right) \\
& =\exp \left(\frac{\log (x)-\log _{s t}(x)}{\nu_{K}(x)} N\right) \eta\left(\operatorname{forget}_{N} D_{s t, \log }(V)\right) \\
& =\eta_{s t, \log } D_{s t, \log }(V) .
\end{aligned}
$$

Example 1.3.13. By Kummer theory any $q \in K^{\times}$defines an extension $V$ of the $\operatorname{Gal}(\bar{K} / K)$-representation $\mathbb{Q}_{p}(0)$ by $\mathbb{Q}_{p}(1)$. This in turn gives via $D_{s t, \log }$ an extension of $K(0)$ by $K(1)$ in $M T_{K}^{\phi, N}$ :

$$
0 \rightarrow K(1) \rightarrow M \rightarrow K(0) \rightarrow 0,
$$

which may be described as follows. The underlying $K_{0}$-space of $M$ has a basis $e_{0}, e_{1}$ such that the following conditions are satisfied:

(1) the action of $\phi$ is given by $\phi\left(e_{i}\right)=p^{-i} e_{i}$, for $i=0,1$,

(2) $e_{1}$ is the image of $1 \in K(1)$,

(3) $e_{0}$ maps to $1 \in K(0)$.

The filtration is given by $F^{-1} M_{K}=M_{K}, F^{0} M_{K}=K \cdot\left\langle\log (q) e_{1}+e_{0}\right\rangle$ and $F^{1} M_{K}=$ 0 . Finally $N$ is given by $N e_{0}=-\nu_{K}(q) \cdot e_{1}$ and $N e_{1}=0$. Then we easily compute

$$
\eta_{s t}(V)=\left(\begin{array}{cc}
1 & 0 \\
\frac{-\nu_{K}(q)\left(\log (x)-\log _{s t}(x)\right)}{\nu_{K}(x)} & 1
\end{array}\right) \cdot\left(\begin{array}{cc}
1 & 0 \\
\log (q) & 1
\end{array}\right)
$$


for the obvious basis of

$$
\tau(V)=\operatorname{Hom}(\mathbb{Q}(0), \mathbb{Q}(0)) \oplus \operatorname{Hom}(\mathbb{Q}(1), \mathbb{Q}(1)),
$$

and every $x \in K_{0} \backslash \mathcal{O}_{K_{0}}^{\times}$, or, equivalently, for every $x \in K \backslash \mathcal{O}_{K}^{\times}$. If $\nu_{K}(q) \neq 0$, then we can take $q=x$ in order to see that

$$
\eta_{s t}(V)=\left(\begin{array}{cc}
1 & 0 \\
\log _{s t}(q) & 1
\end{array}\right)
$$

holds for all $q \in K^{\times}$.

\section{Mixed Tate motives over a number field and logarithmic points}

\subsection{Mixed Tate motives.}

2.1.1. Let $E$ be a number field and $S$ a set of finite places. Let $\mathcal{O}$ be the ring of integers of $E$, and $|\operatorname{Spec}(\mathcal{O})|$ the maximal spectrum of $\mathcal{O}$. We denote by

$$
\mathcal{O}_{S}:=\bigcap_{x \in \operatorname{Spec}(\mathcal{O}) \backslash S} \mathcal{O}_{x}
$$

the ring of $S$-integers of $E$; the elements of $\mathcal{O}_{S}$ are integral outside of $S$. We will be mainly interested in two cases for $S$. In the first case, we have $S=|\operatorname{Spec}(\mathcal{O})|$ and $\mathcal{O}_{S}=E$. In the second case, we have $S=|\operatorname{Spec}(\mathcal{O})| \backslash\{x\}$, for a point $x \in|\operatorname{Spec}(\mathcal{O})|$, and $\mathcal{O}_{S}=\mathcal{O}_{x}$ is the local ring at $x$.

2.1.2. Deligne and Goncharov defined in $[3,1.6]$ an abelian category of mixed Tate motives $M T\left(\mathcal{O}_{S}\right)$. By definition it is the full subcategory of $M T(E)$ consisting of objects which are unramified outside $S$ in the following sense. Let $x \in|\operatorname{Spec}(\mathcal{O})|$ be a point lying over a prime $p$; then we say that $M \in M T(E)$ is unramified at $x$ if for all primes $\ell \neq p$ the corresponding Galois representation $M_{\ell}$ is unramified at $x$, i.e., the inertia subgroup $I_{x}$ (which is only well-defined up to conjugation) acts trivially at $M_{\ell}$ [3, Proposition 1.8].

2.1.3. For extensions of Tate objects we know that:

$$
\begin{aligned}
& \operatorname{Ext}_{M T\left(\mathcal{O}_{S}\right)}^{1}(\mathbb{Q}(0), \mathbb{Q}(1))=\mathcal{O}_{S}^{\times} \otimes \mathbb{Q}, \\
& \operatorname{Ext}_{M T\left(\mathcal{O}_{S}\right)}^{1}(\mathbb{Q}(0), \mathbb{Q}(n))= \begin{cases}0, & \text { if } n \leq 0, \\
\operatorname{Ext}_{M T(E)}^{1}(\mathbb{Q}(0), \mathbb{Q}(n)), & \text { if } n \neq 1,\end{cases} \\
& \operatorname{Ext}_{M T\left(\mathcal{O}_{S}\right)}^{2}(\mathbb{Q}(0), \mathbb{Q}(n))=0, \quad \text { for all } n \in \mathbb{Z},
\end{aligned}
$$

(see [3, Proposition 1.9]).

2.1.4. Every object of $M T\left(\mathcal{O}_{S}\right)$ comes equipped with a finite increasing functorial weight filtration, indexed by even integers. For all $n \in \mathbb{Z}$ the graded pieces $\operatorname{gr}_{2 n}^{W}(M)$ are sums of copies of $\mathbb{Q}(-n)$.

In view of $[3,1.1]$ the $\otimes$-functor

$$
\begin{aligned}
\omega: M T\left(\mathcal{O}_{S}\right) & \rightarrow(\mathbb{Q} \text {-vector spaces }), \\
\omega(M): & =\bigoplus_{n \in \mathbb{Z}} \operatorname{Hom}\left(\mathbb{Q}(n), \operatorname{gr}_{-2 n}^{W}(M)\right),
\end{aligned}
$$


is a fibre functor, therefore $M T\left(\mathcal{O}_{S}\right)$ is a Tannaka category. We denote by $G_{S, \omega}$ the group scheme of $\otimes$-automorphisms of $\omega$. By $[3,2.1]$ we can write $G_{S, \omega}$ as a semi-direct product:

$$
G_{S, \omega}=\mathbb{G}_{m} \ltimes U_{S, \omega},
$$

where $U_{S, \omega}$ is a unipotent group and $G_{S, \omega} \rightarrow \mathbb{G}_{m}$ is induced by the obvious grading of $\omega$. If $S=|\operatorname{Spec}(\mathcal{O})|$ then we simply write $G_{\omega}=G_{S, \omega}$.

2.1.5. Functor to $p$-adic representations. Let $x \in|\operatorname{Spec}(\mathcal{O})|$ be a point lying over a prime $p$. Let $K=E_{x}$ be the completion of $E$ at the place $x$. Choose algebraic closures $\bar{E}, \bar{K}$, and an embedding $\iota: \bar{E} \rightarrow \bar{K}$.

To $M \in M T(E)$ we can attach a Galois representation $M_{p}$ of $\operatorname{Gal}(\bar{E} / E)$ with coefficients in $\mathbb{Q}_{p}$, which is called the $p$-adic realization of $M$. By using $\iota$, we get a continuous homomorphism

$$
\operatorname{Gal}(\bar{K} / K) \rightarrow \operatorname{Gal}(\bar{E} / E),
$$

and we can restrict $M_{p}$ in order to obtain a $p$-adic representation $M_{\iota, p}$ of $\operatorname{Gal}(\bar{K} / K)$.

Proposition 2.1.6. The assignment $M \mapsto M_{\iota, p}$ defines a functor

$$
(.)_{\iota, p}: M T(E) \rightarrow M T_{G_{K}} .
$$

See Definition 1.3.7 for $M T_{G_{K}}$.

Proof. The $p$-adic realization is functorial. Thus, we only need to show that $M_{\iota, p} \in$ $M T_{G_{K}}$, which follows immediately from the existence of the weight filtration of $M$ and Definition 1.3.7.

The set $\left\{\iota: \bar{E} \rightarrow \bar{E}_{x}\right\}$ of embeddings over $E$ is a torsor under the Galois group $\operatorname{Gal}(\bar{E} / E)$, and for every $g \in \operatorname{Gal}(\bar{E} / E)$ there is a natural transformation:

$$
\alpha_{g}:(.)_{\iota, p} \stackrel{\cong}{\longrightarrow}(.)_{\llcorner\circ g, p} \text {. }
$$

Lemma 2.1.7. For the fibre functor $\tau$ (defined in (1.3.8)) and the fibre functor $\omega$ defined in (2.1.1) we have a canonical isomorphism

$$
\tau \circ(.)_{\iota, p} \cong \omega \otimes_{\mathbb{Q}} \mathbb{Q}_{p} .
$$

For every $g \in \operatorname{Gal}(\bar{E} / E)$, the diagram

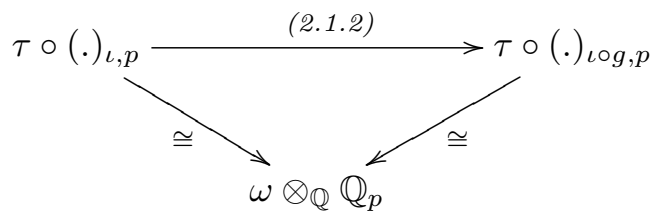

is commutative.

Proof. Straightforward. 
2.1.8. Recall that we have constructed a $K_{s t}$-valued $\eta_{s t}$ of $\underline{\mathrm{Aut}}^{\otimes} \tau$ (Lemma 1.3.12).

Proposition 2.1.9. For every embedding $\iota, \eta_{x}:=\eta_{s t} \circ(.)_{\iota, p}$ defines a $K_{\text {st }}$-valued point of $\underline{\mathrm{Aut}}_{M T(E)}^{\otimes} \omega$ which is independent of the choice of $\iota$.

Proof. Since $\tau \circ(.)_{\iota, p}=\omega \otimes_{\mathbb{Q}} \mathbb{Q}_{p}$ by Lemma 2.1.7, $\eta_{s t} \circ(.)_{\iota, p}$ is a $K_{s t}$-valued point of $\underline{\mathrm{Aut}}^{\otimes} \omega$.

The independence of the choice of $\iota$ follows from the commutative diagram

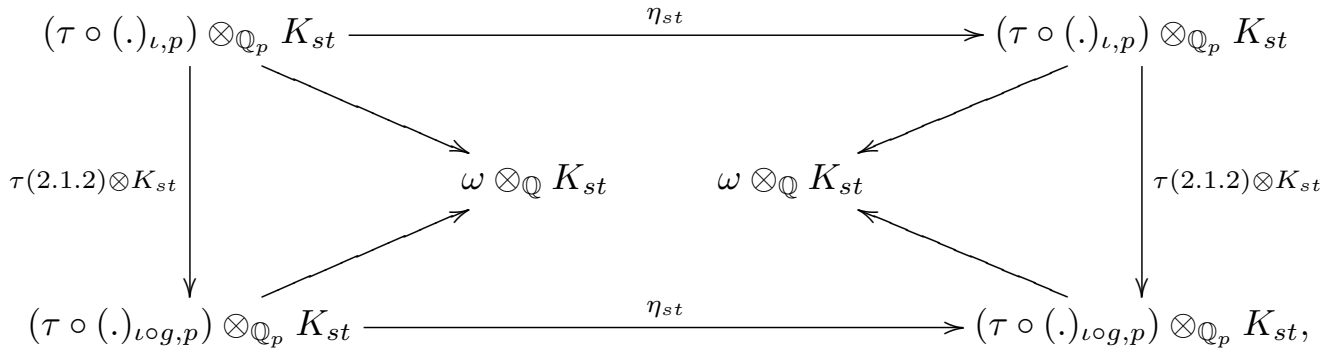

where the triangles are commutative by Lemma 2.1.7, and the square is commutative because $\eta_{s t}$ is functorial.

\subsection{Crystalline characterization of unramified motives.}

2.2.1. Let $E$ be a number field, and let $M$ be a mixed Tate motive over $E$, i.e., an object in $M T(E)$. Let $\nu$ be a finite place of $E, M$ is unramified at $\nu[3$, Definition 1.4 , Section 1.7] if the coaction $[3,(1.2 .2)]$

$$
e_{M}: \omega(M) \rightarrow \operatorname{Ext}^{1}(\mathbb{Q}(0), \mathbb{Q}(1)) \otimes \omega(M)
$$

of $\operatorname{Ext}^{1}(\mathbb{Q}(0), \mathbb{Q}(1))=E^{\times} \otimes_{\mathbb{Z}} \mathbb{Q}$ on $\omega(M)$ factors through a coaction of $\mathcal{O}_{\nu}^{\times} \otimes_{\mathbb{Z}} \mathbb{Q}$.

2.2.2. Recall from Proposition 2.1.6 that $M_{\iota, p}$ is a mixed Tate Galois representation of $G_{K}=\operatorname{Gal}(\bar{K} / K)$ for the completion $K=E_{\nu}$ at $\nu$. In particular, $M_{\iota, p}$ is semistable (Proposition 1.3.8). In the following we will simply write $M_{p}=M_{p, \iota}$. We call $M_{p}$ crystalline if the monodromy operator $N$ of $D_{s t}\left(M_{p}\right)$ is trivial, or equivalently if

$$
\left(B_{\text {cris }} \otimes_{\mathbb{Q}_{p}} M_{p}\right)^{G_{K}} \rightarrow\left(B_{s t} \otimes_{\mathbb{Q}_{p}} M_{p}\right)^{G_{K}}
$$

is an isomorphism.

Theorem 2.2.3. Let $M$ be a mixed Tate motive over $E$ and $\nu$ a finite place of $E$. Then $M$ is unramified at $\nu$ if and only if $M_{p}$ is crystalline.

Proof. First note that the statement that $M$ is unramified at $\nu$ is equivalent to the statement that for every subquotient $N$ of $M$ which is of the form

$$
0 \rightarrow \mathbb{Q}(n+1) \rightarrow N \rightarrow \mathbb{Q}(n) \rightarrow 0,
$$

for some $n$, the extension class $\operatorname{Ext}^{1}(\mathbb{Q}(n), \mathbb{Q}(n+1))=\operatorname{Ext}^{1}(\mathbb{Q}(0), \mathbb{Q}(1))=E^{\times} \otimes \mathbb{Q}$ lies in $\mathcal{O}_{\nu}^{\times} \otimes_{\mathbb{Z}} \mathbb{Q}[3$, Section 1.4].

Also in the category of $p$-adic representations of a $p$-adic field $K$, a representation in $\operatorname{Ext}_{G_{K}}^{1}\left(\mathbb{Q}_{p}(0), \mathbb{Q}_{p}(1)\right)$ that is associated to some $q \in K^{\times} \otimes \mathbb{Q} \subseteq \lim _{n}\left(K^{\times} /\left(K^{\times}\right)^{p^{n}}\right) \otimes$ $\mathbb{Q}=\operatorname{Ext}_{G_{K}}^{1}\left(\mathbb{Q}_{p}(0), \mathbb{Q}_{p}(1)\right)$ is crystalline if and only if $q \in \mathcal{O}_{K}^{\times} \otimes \mathbb{Q}[6$, Example 2.3.2]. 
First, suppose that $M_{p}$ is crystalline, then every subquotient of $M_{p}$ is crystalline. So in order to prove that $M$ is unramified at $\nu$ we may assume that $M=N$, where $N$ is as above with $n=0$ (after Tate twist). Therefore we have an extension in $\operatorname{Ext}^{1}(\mathbb{Q}(0), \mathbb{Q}(1))$, defined by some $q \in E^{\times} \otimes_{\mathbb{Z}} \mathbb{Q}$, whose $p$-adic realization is crystalline at $\nu$. Then the above remark implies that the image of $q$ in $E_{\nu}^{\times} \otimes \mathbb{Q}$ lies in $\hat{\mathcal{O}}_{\nu}^{\times} \otimes \mathbb{Q}$, hence $q \in \mathcal{O}_{\nu}^{\times} \otimes \mathbb{Q}$ and $M$ is unramified at $\nu$.

Suppose conversely that $M$ is unramified at $\nu$. We have to show that the monodromy operator $N$ on $D_{s t}\left(M_{p}\right)=: D(M)$ vanishes. Note that $N$ maps the slope $\lambda$ piece of $D(M)$ to the slope $\lambda-1$ piece. Therefore, if $N$ is non-zero on $D(M)$ then there exists an $n$ such that $N$ is non-zero on

$$
D\left(W_{2 n} M_{p} / W_{2 n-4} M_{p}\right)=D\left(\left(W_{2 n} M / W_{2 n-4} M\right)_{p}\right) .
$$

Replacing $M$ by $\left(W_{2 n} M / W_{2 n-4} M\right) \otimes \mathbb{Q}(n)$ we may assume that $M$ is defined by a class in $\operatorname{Ext}^{1}\left(\mathbb{Q}(0)^{\oplus r}, \mathbb{Q}(1)^{\oplus s}\right)=\operatorname{Ext}^{1}(\mathbb{Q}(0), \mathbb{Q}(1))^{\oplus r s}, M$ is unramified, and $N$ is non-zero on $D(M)$. By passing to a subquotient we may further assume that $r=s=1$. This gives an extension in $\operatorname{Ext}^{1}(\mathbb{Q}(0), \mathbb{Q}(1))$, which is unramified at $\nu$ (and hence defined by some $q \in \mathcal{O}_{\nu}^{\times} \otimes \mathbb{Q}$ ) and whose $p$-adic realization is not crystalline at $\nu$. This is a contradiction.

2.2.4. Recall the notation of Section 2.1.1. Let $x \in|\operatorname{Spec}(\mathcal{O})|$ be a point; in the following we will work with $S=|\operatorname{Spec}(\mathcal{O})| \backslash\{x\}$, thus $\mathcal{O}_{S}=\mathcal{O}_{x}$.

Let $p$ be the prime lying under $x$. In view of Theorem 2.2.3, we know that $\operatorname{MT}\left(\mathcal{O}_{x}\right)$ is the full subcategory of $M T(E)$ consisting of motives $M$ such that the $p$-adic realization $M_{p}$ is crystalline at $x$.

We denote by $G_{x}$ the group scheme of $\otimes$-automorphisms of the fibre functor (see $(2.1 .1))$

$$
\omega: M T\left(\mathcal{O}_{x}\right) \rightarrow(\mathbb{Q} \text {-vector spaces }) .
$$

The group scheme $G_{x}$ is a quotient of $G_{\omega}=\operatorname{Aut}_{M T(E)}^{\otimes} \omega$.

Lemma 2.2.5. The morphism $\operatorname{Spec}\left(E_{x, s t}\right) \stackrel{\eta_{x}}{\longrightarrow} G_{\omega} \rightarrow G_{x}$ factors through the structure morphism $\operatorname{Spec}\left(E_{x, s t}\right) \rightarrow \operatorname{Spec}\left(E_{x}\right)$ and thus defines a point $\eta_{x}^{u r} \in G_{x}\left(E_{x}\right)$.

Proof. The point $\eta_{x}$ was defined in Proposition 2.1.9. If $M \in M T\left(\mathcal{O}_{x}\right)$ then $D_{s t}\left(M_{\iota, p}\right)$ has vanishing monodromy operator $N$ and $\eta_{s t}\left(M_{\iota, p}\right)=\eta_{s t, \log } D_{s t}\left(M_{\iota, p}\right)$ takes values in $E_{x}$ by Definition 1.3.10.

\subsection{Main theorem.}

2.3.1. Let $x \in|\operatorname{Spec}(\mathcal{O})|$ and let $E_{x}$ be the completion of $E$ at $x$. Bloch and Kato [1, Definition 3.10] define an exponential map

$$
\exp : E_{x} \rightarrow \operatorname{Ext}^{1}\left(\mathbb{Q}_{p}(0), \mathbb{Q}_{p}(n)\right), \quad \text { for all } n \geq 1,
$$

where $\operatorname{Ext}^{1}$ is computed in the category of $p$-adic representation of $\operatorname{Gal}\left(\bar{E}_{x} / E_{x}\right)$. Note that, in fact, the image of the exponential map lies among the crystalline representations $\operatorname{Ext}_{\text {crys }}^{1}\left(\mathbb{Q}_{p}(0), \mathbb{Q}_{p}(n)\right)[1$, Example 3.9]. Via $p$-adic Hodge theory, we obtain a map

$$
E_{x} \rightarrow \operatorname{Ext}_{\text {crys }}^{1}\left(\mathbb{Q}_{p}(0), \mathbb{Q}_{p}(n)\right) \cong \operatorname{Ext}_{M T_{E_{x}}^{\phi}}^{1}\left(E_{x}(0), E_{x}(n)\right),
$$

which, by abuse of notation, will also be called the Bloch-Kato exponential map. 
2.3.2. For an extension $M \in \operatorname{Ext}_{M T\left(\mathcal{O}_{x}\right)}^{1}(\mathbb{Q}(0), \mathbb{Q}(n))$ with $n \geq 1$, there are natural maps $v_{0}: \mathbb{Q} \rightarrow \omega(M)$ and $f_{n}: \omega(M) \rightarrow \mathbb{Q}$ defined as follows. By definition, there are isomorphisms $\alpha: \mathbb{Q}(n) \rightarrow \operatorname{gr}_{-2 n}^{W} M$ and $\beta: \operatorname{gr}_{0}^{W} M \rightarrow \mathbb{Q}(0)$; we define

$$
\begin{aligned}
& v_{0}: \mathbb{Q}=\operatorname{Hom}(\mathbb{Q}(0), \mathbb{Q}(0)) \stackrel{\beta^{-1}}{\longrightarrow} \omega_{0}(M) \rightarrow \omega(M), \\
& f_{n}: \omega(M) \rightarrow \omega_{n}(M) \stackrel{\alpha^{-1}}{\longrightarrow} \operatorname{Hom}(\mathbb{Q}(n), \mathbb{Q}(n))=\mathbb{Q} .
\end{aligned}
$$

Given a point $s: \operatorname{Spec}(L) \rightarrow G_{x}$, we get $s(M): \omega(M) \otimes L \rightarrow \omega(M) \otimes L$. This gives an element of $L$ as follows:

$$
M(s):=f_{n}\left(s(M)\left(v_{0}(1)\right)\right) \in L .
$$

Theorem 2.3.3. Let $E$ be a number field and $\mathcal{O}$ be the ring of integers. Let $x \in$ $|\operatorname{Spec}(\mathcal{O})|$ be a closed point over a prime p. For the Tannaka category $\left(\operatorname{MT}\left(\mathcal{O}_{x}\right), \omega\right)$ of mixed Tate motives we denote by $G_{x}$ the group scheme of $\otimes$-automorphisms of $\omega$. For all $n \geq 1$, the map

$$
\operatorname{Ext}_{M T\left(\mathcal{O}_{x}\right)}^{1}(\mathbb{Q}(0), \mathbb{Q}(n)) \rightarrow E_{x}, \quad M \mapsto M\left(\eta_{x}^{u r}\right),
$$

induced by $\eta_{x}^{u r} \in G_{x}\left(E_{x}\right)$ (see Lemma 2.2.5), is the composition of the p-adic realization

$$
\operatorname{Ext}_{M T\left(\mathcal{O}_{x}\right)}^{1}(\mathbb{Q}(0), \mathbb{Q}(n)) \rightarrow \operatorname{Ext}_{\text {crys }}^{1}\left(\mathbb{Q}_{p}(0), \mathbb{Q}_{p}(n)\right)
$$

and the inverse of the Bloch-Kato exponential map (2.3.1).

Proof. Let us prove that evaluation at the point $\eta_{x}^{u r}$ has the desired compatibility with the Bloch-Kato exponential map (see (2.3.2))

$$
\exp : E_{x} \rightarrow \operatorname{Ext}_{\text {crys }}^{1}\left(\mathbb{Q}_{p}(0), \mathbb{Q}_{p}(n)\right) \stackrel{\cong}{\longrightarrow} \operatorname{Ext}_{M T_{E_{x}}}^{1}\left(E_{x}(0), E_{x}(n)\right) \text {. }
$$

For this we need to recall the construction of the exponential map. For the rest of the proof let $K:=E_{x}$. First there is an exact sequence [1, Proposition 1.17]:

$$
0 \rightarrow \mathbb{Q}_{p} \rightarrow B_{\text {crys }}^{\varphi=1} \oplus B_{d R}^{+} \rightarrow B_{d R} \rightarrow 0
$$

where the first map sends $x$ to $(x, x)$ and the second one sends $(x, y)$ to $x-y$.

For $n \geq 1$, the Bloch-Kato construction gives a map

$$
K=\left(\mathbb{Q}_{p}(n) \otimes B_{d R}\right)^{G_{K}} \rightarrow \operatorname{Ext}_{\text {crys }}^{1}\left(\mathbb{Q}_{p}(0), \mathbb{Q}_{p}(n)\right) .
$$

This map is obtained as follows. First tensor the above exact sequence with $\mathbb{Q}_{p}(n)$ :

$$
0 \rightarrow \mathbb{Q}_{p}(n) \rightarrow\left(\mathbb{Q}_{p}(n) \otimes B_{\text {crys }}^{\varphi=1}\right) \oplus\left(\mathbb{Q}_{p}(n) \otimes B_{d R}^{+}\right) \rightarrow \mathbb{Q}_{p}(n) \otimes B_{d R} \rightarrow 0 .
$$

Then an element $a$ in $K(n)=\left(\mathbb{Q}_{p}(n) \otimes B_{d R}\right)^{G_{K}}$ gives a map $\mathbb{Q}_{p} \rightarrow \mathbb{Q}_{p}(n) \otimes B_{d R}$, pulling back the above exact sequence via this map gives the extension we were looking for.

More explicitly, for $a \in K$ the extension constructed above is:

$$
0 \rightarrow V_{n} \rightarrow V \rightarrow V_{0} \rightarrow 0
$$

where $V_{0}=\mathbb{Q}_{p} \cdot t^{n} \otimes a t^{-n}, V_{n}=\mathbb{Q}_{p} \cdot t^{n}$, and $V$ is a two-dimensional representation of $G_{K}$ with basis which can be described as follows. By the exact sequence (2.3.3), there exists $x \in B_{\text {crys }}^{\varphi=1}$ and $y \in B_{d R}^{+}$such that $a t^{-n}=x-y$. Then $V$ has basis $\left\{\left(t^{n} \otimes x, t^{n} \otimes y\right),\left(t^{n} \otimes 1, t^{n} \otimes 1\right)\right\}$. For $\sigma \in G_{K}$,

$$
\sigma\left(t^{n} \otimes x, t^{n} \otimes y\right)=\left(t^{n} \otimes x, t^{n} \otimes y\right)+\gamma(\sigma)\left(t^{n} \otimes 1, t^{n} \otimes 1\right),
$$


for some $\gamma(\sigma) \in \mathbb{Q}_{p}$. Therefore

$$
\chi_{\text {сус }}(\sigma)^{n} \sigma(x)=x+\gamma(\sigma)
$$

and

$$
\chi_{\mathrm{cyc}}(\sigma)^{n} \sigma(y)=y+\gamma(\sigma) .
$$

Let us now try to find what this extension corresponds to after we apply the functor $\left(\cdot \otimes B_{\text {crys }}\right)^{G_{K}}$. First note that $\left(V \otimes B_{\text {crys }}\right)^{G_{K}}$ has basis

$$
e_{n}:=\left(t^{n} \otimes 1, t^{n} \otimes 1\right) \otimes t^{-n}
$$

and

$$
e_{0}:=\left(t^{n} \otimes x, t^{n} \otimes y\right) \otimes 1-\left(t^{n} \otimes 1, t^{n} \otimes 1\right) \otimes x .
$$

That $e_{n}$ is invariant under the Galois action is clear. In order to see that $e_{0}$ is $G_{K}$ invariant let $\sigma \in G_{K}$. Then

$$
\begin{aligned}
\sigma\left(e_{0}\right)= & \left(t^{n} \otimes(x+\gamma(\sigma)), t^{n} \otimes(y+\gamma(\sigma))\right) \\
& \otimes 1-\left(t^{n} \otimes 1, t^{n} \otimes 1\right) \otimes(x+\gamma(\sigma))=e_{0} .
\end{aligned}
$$

Now note that $\varphi\left(e_{n}\right)=p^{-n} e_{n}$ and $\varphi\left(e_{0}\right)=e_{0}$. Furthermore $e_{n}$ is the image of $1 \in K(n)$ and $e_{0}$ maps to $1 \in K(0)$ in the exact sequence (note that $\mathbb{Q}_{p}(0)$ is identified with $V_{0}$ via the map that sends 1 to $\left.t^{n} \otimes a t^{-n}\right)$ :

$$
0 \rightarrow K(n) \rightarrow\left(V \otimes B_{\text {crys }}\right)^{G_{K}} \rightarrow K(0) \rightarrow 0 .
$$

Therefore, in order to compare Bloch-Kato's construction we need only compute the filtration on $\left(V \otimes B_{\text {crys }}\right)^{G_{K}} \otimes_{K_{0}} K$. So we need to compute the 0 th piece of the filtration on $\left(V \otimes B_{d R}\right)^{G_{K}}$.

We claim that $a e_{n}+e_{0} \in F i l^{0}\left(V \otimes B_{d R}\right)^{G_{K}}$. This follows immediately from

$$
a e_{n}+e_{0}=\left(t^{n} \otimes x, t^{n} \otimes y\right) \otimes 1-\left(t^{n} \otimes 1, t^{n} \otimes 1\right) \otimes y,
$$

and the fact that $y \in B_{d R}^{+}$.

In order to complete the proof, let us start with $M$ in $\operatorname{Ext}_{M T\left(\mathcal{O}_{x}\right)}^{1}(\mathbb{Q}(0), \mathbb{Q}(n))$. The $p$-adic realization of $M$ gives an element $M_{x}$ in $\operatorname{Ext}_{M T_{E_{x}}^{\phi}}^{1}\left(E_{x}(0), E_{x}(n)\right)$. Then $M_{x}=\exp (a)$, for some $a \in E_{x}$. On the other hand, by the computation above $\exp (a)$ is the extension in $\operatorname{Ext}_{M T_{E_{x}}^{\phi}}^{1}\left(E_{x}(0), E_{x}(n)\right)$, whose 0th filtration is given by $\left\langle a \cdot e_{n}+e_{0}\right\rangle$, where $\phi\left(e_{i}\right)=p^{-i} e_{i}$ and $e_{i}$ is the element 1 in $E_{x}(i)$, for $i=0, n$ (cf. Proposition 1.2.6). In view of the proof of Proposition 1.2.6 we see that $f_{n}\left(\eta_{x}^{u r}(M) v_{0}(1)\right)=$ $f\left(\eta\left(M_{x}, \phi, F\right)(v(1))\right)=a$. This completes the proof.

\section{Acknowledgments}

The first author has been supported by the SFB/TR 45 "Periods, moduli spaces and arithmetic of algebraic varieties". The second author was supported by Tüba-Gebip and Tübitak grant 109T674. 


\section{References}

[1] S. Bloch and K. Kato, L-functions and Tamagawa numbers of motives, in The Grothendieck Festschrift, Vol. I, Vol. 86 of Progress in Mathematics, 333-400, Birkhäuser Boston, Boston, MA (1990).

[2] P. Colmez and J.-M. Fontaine, Construction des représentations p-adiques semi-stables, Invent. Math. 140(1) (2000), 1-43.

[3] P. Deligne and A. B. Goncharov, Groupes fondamentaux motiviques de Tate mixte, Ann. Sci. École Norm. Sup. (4) 38(1) (2005), 1-56.

[4] J. I. Manin, Theory of commutative formal groups over fields of finite characteristic, Uspehi Mat. Nauk 18(6) (1963), (114), 3-90.

[5] J. Nekováŕ, On p-adic height pairings, in Séminaire de Théorie des Nombres, Paris, 1990-91, Vol. 108 of Progr. Math., 127-202, Birkhäuser Boston, Boston, MA (1993).

[6] T. Tsuji, Semi-stable conjecture of Fontaine-Jannsen: a survey, Astérisque (2002), no. 279, 323370. Cohomologies $p$-adiques et applications arithmétiques, II.

Fachbereich Mathematik, Universität Duisburg-Essen, 45117 Essen, Germany

E-mail address: a.chatzistamatiou@uni-due.de

Mathematics Department, KoÇ University, 34450, Istanbul, Turkey

E-mail address: sunver@ku.edu.tr 